\title{
Cobertura de atención de enfermería y su impacto en la mortalidad en México, 2007
}

\author{
Nursing care coverage and its impact \\ on mortality in Mexico, 2007.
}

Mtra. María Dolores Zarza Arizmendi•, Mtra. Rosa Amarilis Zárate Grajales •., Mtro. Rey Arturo Salcedo

Álvarez ..., Lic. Blanca Consuelo González Caamaño .... y Dr.. Guillermo Fajardo-Ortiz .....

\section{Resumen}

Introducción: El estudio de la mortalidad contribuye para conocer la situación de la salud de la población e indica los retos en las políticas públicas para disminuir las muertes prematuras y/o evitables. Objetivo: Identificar como impacta el indicador de cobertura de atención de enfermería en la mortalidad infantil, perinatal, materna y en menores de cinco años. Metodología: Estudio transversal y analítico donde se utilizó información oficial de recursos humanos de enfermería y mortalidad por entidad federativa en México 2007, a partir del método de "Componentes Principales" se construyó el Índice Global de Mortalidad y se buscó asociación causal. Resultados: En México la cobertura de enfermería es de 2.11 enfermeras por cada 1,000 habitantes, sin embargo, al interior del país se observan diferencias de hasta tres veces para este indicador. El ejercicio de regresión lineal simple demuestra asociación estadísticamente significativa ya que conforme se incrementa la cobertura de enfermería se disminuye la mortalidad. Discusión: Definitivamente, la cobertura de enfermería no es el único factor que determina la mortalidad. Sin embargo, en estudios publicados en los últimos años se identifica que el cuidado de enfermería sí incrementa los niveles de salud de la población, retrasa la edad de la muerte y disminuye las muertes tempranas y evitables. Conclusiones: El estudio de la mortalidad temprana y/ o evitable y su relación con la cobertura de enfermería son sólo un factor para la mejor comprensión del impacto potencial que puede llegar a tener la presencia del profesional de enfermería en el perfil de salud y la mortalidad en la población.

- Profesor de Carrera Asociado "C", Secretaria General, ENEO-UnAm... Profesor de Carrera Asociado "C", Jefe de la Unidad de Posgrado e Investigación y Directora Adjunta del Centro Colaborador de la OPS. ENEOUNAM. ...Profesor de Carrera Asociado “B”, Unidad de Investigación, ENEO-UNAM. .... Profesor de la ENEOUnAM, División de Estudios Profesionales. ..... Profesor de Carrera Titular "C", Facultad de Medicina, unAM. Correspondencia:mdz55@hotmail.com • rasalced@hotmail.com

RECIBIDO 29 DE MAYO 2010-09 ENVIADO 1 DE JUNIO 2010-09

ACEPTADO 28 DE JUNIO 2010 
Introduction: The mortality study contributes to understand the health status of the population and it indicates the challenges in public policy to reduce premature and / or preventable death. Objective: To identify how nursing care coverage indicator impacts on infant mortality, perinatal mortality, maternal mortality and under five years old mortality. Methodology: Transversal and analytic study where official information of Nursing Human Resources and mortality by federal entity in Mexico 2007 was used, from the method of "Main Components" the "Global Mortality Index "was built and causal association was looked for. Results: In Mexico nursing coverage is 2.11 nurses per 1,000 inhabitants, however, within the country differences are observed of up to three times for this indicator. The exercise of simple linear regression shows statistically significant association since according to increasing nursing coverage mortality decreases. Discussion: Definitely, nursing coverage is not the only factor determining mortality. However, in studies published in recent years it is possible to identify that nursing care does increase the levels of population's health, delays age of death and reduces early and avoidable deaths. Conclusions: The study of early and / or preventable mortality and its relation to nursing coverage are only one factor for a better understanding of the potential impact that the presence of professional nurses can have in the health profile and mortality among population.

\section{INTRODUCCIÓN}

La Reforma del Sector Salud en México, incluye el desarrollo de estrategias para analizar las características de la atención, del ambiente organizacional y de la capacidad de los gestores para identificar el número y tipo de profesionales que contribuyan a mejorar las condiciones de salud de la población, para brindar mejores coberturas y mayor calidad en la prestación de los servicios de salud.

El siguiente artículo pretende contribuir al análisis de la situación de enfermería y su vinculación con la mortalidad en nuestro país. Se sabe que es complejo contar con la dotación ideal de personal, las características de la composición, distribución y suficiencia de los recursos humanos en una orga- nización de salud no siempre son las adecuadas. Sin embargo, los responsables de gestión durante la toma de decisiones, deberán incluir estos conceptos en el análisis de los recursos humanos para lograr el cumplimiento de metas programadas.

La Organización Panamericana de la Salud (OPS) en el documento "Dotación de recursos humanos en salud nuevas perspectivas de análisis", propone ${ }^{1}$ que la gestión de los recursos humanos se realice desde tres áreas: a) enfatizando en la obtención de la información que permita definir la cantidad de personal necesario para alcanzar la cobertura y satisfacer las necesidades en salud y con ello fijar los estándares o criterios nacionales que permitan la adecuación y prestación de ser- vicios requeridos por la población; b) incluyendo la calidad de la atención a partir de los estándares definidos y el establecimiento de metas que permitan mejorar gradualmente la prestación de los servicios con indicadores de calidad; y c) formalizando la relación entre la gestión y el trabajo específico de enfermería, con el tiempo destinado a las acciones prioritarias a partir de los procesos previamente establecidos.

De acuerdo a estos tres lineamientos, las necesidades de cobertura de atención y los requerimientos de personal de enfermería son el enfoque del presente artículo para abordar el análisis desde el punto de vista de las necesidades de salud de la población, la dotación de personal de enfermería y el impacto que éste tiene en la mortalidad. 


\section{RECURSOS HUMANOS EN ENFERMERÍA Y MORTALIDAD}

Las características de mortalidad de una población describen no solamente la situación de la salud de la población, si no también indican los retos que ésta representa para las instituciones de salud, en cuestión de políticas públicas encaminadas a disminuir las muertes prematuras y/o evitables, tal es el caso de las infantiles y maternas. El análisis multifactorial de las causas de muerte ayuda a mejorar las condiciones de vida, los riesgos para la salud y el efecto real de las políticas de salud vigentes. ${ }^{2}$

En este sentido, el profesional de enfermería es parte fundamental del desarrollo de los sistemas y servicios de salud, consecuentemente, es necesario contar con suficiencia de recursos humanos calificados para satisfacer las necesidades de cuidado a la salud de la población. Desde luego sería ilógico afirmar que la cobertura de atención de enfermería es el único factor que determina el perfil de mortalidad, sabemos que no es así, sin embargo, el cuidado de enfermería sí coadyuva al nivel de salud de la población.

De acuerdo con Suzanne Kérouac los cuidados enfermeros, se han convertido en complejos y diversificados, por lo que en la actualidad se requiere desarrollar competencias en conocimientos científicos y utilización de métodos. ${ }^{3}$ Así mismo, el Consejo Internacional de Enfermeras (ICE) define a la enfermería como la disciplina encargada de proporcionar los "Cuidados autónomos y en colaboración, que se prestan a las personas de todas las edades, familias, grupos y comunidades, enfermos o sanos, en todos los contextos, e incluye la promoción de la salud, la prevención de la enfermedad, y los cuidados de los enfermos, discapacitados, y personas moribundas. Funciones esenciales de la enfermería son la defensa de los derechos de los pacientes, el fomento de un entorno seguro, la investigación, la participación en políticas de salud y en la gestión de los pacientes y los sistemas de salud, así como la formación de recursos humanos".

Ante tal situación, la evaluación de los recursos para la atención a la salud debe hacerse a través de indicadores de estructura, proceso y resultado. Para el caso particular de los "Recursos" de acuerdo con Donabedian, éstos se refieren a la cuantificación de las "unidades que producen servicio y se encuentran en tiempo y lugar," ${ }^{5}$ tal sería el caso de la cobertura de atención de enfermería, misma que considera a la población potencial, es decir aquella a la que se puede proporcionar atención dada la infraestructura existente y la población usuaria. ${ }^{6}$

Criterios internacionales sobre cobertura de servicios de atención en enfermería, consideran que un país que cuenta con menos de 20 enfermeras por cada 10,000 habitantes se clasifica en nivel bajo; más de 20 y menos de 40 se ubica en nivel medio y con más de 40 en nivel alto. Con estos criterios, México presenta dos realidades, si consideramos la cobertura en población con seguridad social se ubica en el nivel medio, pero si se trata de la población sin seguridad social la cobertura esta en un nivel bajo. Con base en el análisis anterior en un estudio realizado por Zárate y Salcedo en el 2007 en México para alcanzar cuando menos un nivel medio de 40 enfermeras por cada 10,000 habitantes, se requerirán otras 227,493 enfermeras, sin embargo, en el caso de que se pretendiera alcanzar el indicador con personal profesional se requeriría de 312,819 enfermeras adicionales. ${ }^{7}$

Estudios realizados en EEUU, demostraron el impacto que tiene la falta de personal cualificado de enfermería en la evolución de la salud los pacientes. ${ }^{8,9}$ En México, aun no tenemos estudios similares, sin embargo, utilizando información de mortalidad del Sistema Nacional de Salud (SNS) mexicano observamos que el rezago en salud guarda una estrecha relación con el número de enfermeras, esta situación se hace evidente en las estadísticas nacionales de mortalidad. ${ }^{7}$

\section{LA MORTALIDAD COMO INDICADOR}

Si bien, la muerte es un fenómeno infranqueable para todos los seres vivos, es deseable que ésta ocurra de manera tardía, por causas naturales y en condiciones de confort, la ocurrencia de muertes a edades tempranas y/o por causas evitables son un aviso de que las políticas de salud son aun perfectibles. De acuerdo con la Ops "Las mortalidades materna e infantil son indicadores de la salud objetiva de una población" ${ }^{10}$

Por consiguiente, la información en salud constituye un elemento fundamental no sólo para conocer el estado de salud de una población, sino además para que, a partir de ésta, se puedan poner en práctica nuevos programas, actualizar los que cumplan con los objetivos propuestos, evaluar las condiciones cambiantes de la salud de 
la población. Sin información veraz y oportuna no se puede diseñar ni llevar a cabo una verdadera política de salud. ${ }^{6}$

En este orden de ideas y para facilitar las comparaciones internacionales, la Organización Mundial de la Salud definió dentro de su sistema de observación y evaluación un conjunto de indicadores globales, entre los que se encuentran las mortalidades: infantil, perinatal, materna y en menores de cinco años. ${ }^{6}$

En términos generales la mortalidad se define como número proporcional de muertes en una población y tiempo determinados, la mortalidad infantil hace referencia a las defunciones ocurridas entre los niños menores de un año de edad; la perinatal abarca las defunciones fetales desde los 1,000 gramos y más de peso hasta los neonatos fallecidos antes de los 7 días de vida con 1,000 gramos o más de peso al nacer; la mortalidad en menores de cinco años se refiere a las muertes del grupo de 1 a 4 años de edad; y la mortalidad materna a los decesos en mujeres embarazadas o dentro de los 42 días siguientes a la terminación del mismo, independientemente de la duración y el sitio del embarazo, debida a cualquier causa relacionada con el embarazo o su atención, pero no por causas accidentales. ${ }^{11,12}$

\section{ÍNDICE GLOBAL DE Mortalidad}

Se entiende por indicador de salud al valor numérico de cualquier cifra absoluta o relativa que refleje una situación determinada y que por comparaciones en el tiempo y en el espacio permita observar los cambios en dicha situación. ${ }^{6}$
El Índice Global de Mortalidad (IGM) es un indicador resumen que los autores de éste artículo proponen como un constructo basado en distintos tipos de mortalidad como son: mortalidad infantil, perinatal, materna y en menores de cinco años. Para tener un valor sustantivo de mortalidad global para cada entidad federativa y pueda ser objeto de priorización.

Su cálculo se realiza a partir del "Método de Componentes Principales" que tiene por objeto reducir las dimensiones de un problema de múltiples variables, aplicando una sucesión de transformaciones lineales a las variables de modo que un subconjunto de ellas concentre la mayor parte de la variabilidad contenida en las variables originales. ${ }^{13}$

Supóngase que se tienen $n$ sujetos y $p$ variables, con estos datos es posible formar una matriz de $n$ renglones por $p$ columnas en la cual existan más sujetos que variables $(n>p)$.

El IGM varía entre 0 y 100 . Su valor expresa la magnitud de la mortalidad, mientras más cercana a 100 es ésta quiere decir que es mayor la mortalidad, en consecuencia se trata de un método de priorización.
$\mathrm{Su}$ formulación matemática es: $\mathrm{IGM}=1 / 4(\mathrm{IMI}+\mathrm{IMP}+\mathrm{IMM}+\mathrm{IM}>5)$

- Donde IGM es el Índice Global de Mortalidad,

- $1 / 4$ representa la fracción proporcional de la centésima,

- IMI es el índice de Mortalidad Infantil,

- IMP es el Índice de Mortalidad Perinatal,

- IMm es el Índice de Mortalidad Materna, y

- $\quad \mathrm{IM}>5$ es el Índice de Mortalidad en menores de cinco años.

Para la construcción de cada uno de los cuatro componentes se utilizó la siguiente fórmula:

$\mathrm{IMI}=\frac{\text { MIi }- \text { MImín }}{\text { MImáx }- \text { MImín }} * 100$

- Donde ImI es el Índice de Mortalidad Infantil, MIi representa el valor que se quiere priorizar,

- MImáx representa el valor máximo de la población con la que se quiere comparar, y

- MImín representa el valor mínimo de la población con la que se quiere comparar.

\section{Matriz para la construcción del Índice Global de Mortalidad}

\begin{tabular}{lllll}
$\begin{array}{l}\text { Indicador } \\
\text { de }\end{array}$ & \multicolumn{3}{c}{ Máximo } & \multicolumn{3}{l}{ Mínimo } \\
Mortalidad & Valor & Estado & Valor & Estado \\
\hline Infantil & 23.3 & Puebla & 8.1 & Nayarit \\
Perinatal & 30.8 & D. F. & 6.1 & Sinaloa \\
Materna & 1.02 & Oaxaca & 0.175 & Aguascalientes \\
$<5$ años & 26.1 & Guerrero & 14.5 & D. F.
\end{tabular}


Ejemplo: Para calcular el IMI que es el Índice de Mortalidad Infantil del Estado de Aguascalientes utilizando la fórmula descrita:

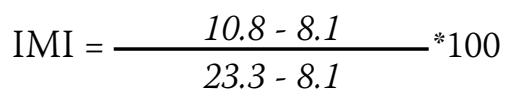

- Donde IMI es el Índice de Mortalidad Infantil,

- MIi representa la tasa de mortalidad infantil de Aguascalientes que es de 10.8 defunciones en niños de 1 a 12 meses de edad por 1,000 NVR,

- MImáx representa la tasa de mortalidad infantil de Puebla que es de 23.3 defunciones en niños de 1 a 12 meses de edad por 1,000 NVR, y MImín representa la tasa de mortalidad infantil de Nayarit que es de 8.1 defunciones en niños de 1 a 12 meses de edad por 1,000 NVR.

En este caso el IMI es de 17.7 para el estado de Aguascalientes. ${ }^{14}$

\section{Objetivo}

Identificar como impacta el indicador de cobertura de atención de enfermería en la mortalidad infantil, perinatal, materna y en menores de cinco años.

\section{Metodología}

Se realizó un estudio de tipo transversal, analítico. Utilizando la información de población total y recursos humanos de enfermería por entidad federativa y se calculó el Índice de Cobertura de Enfermería por 1,000 habitantes.

Únicamente se utilizaron fuentes de información oficial del SNS, con datos actualizados hasta el año 2007, tales como: publicaciones de la Dirección General de Informa- ción en Salud, el Boletín de Información Estadística, el Anuario de Mortalidad y los Indicadores Básicos de Salud en México.

Con base al Índice de Cobertura de Enfermería (ICE) se realizó una distribución cuartilar donde se estratificaron los diferentes subgrupos de entidades federativas según en nivel de cobertura de atención, quedando de la siguiente manera: menor o igual a 1.83 enfermeras por 1,000 habitantes, se determinó como cobertura baja; de 1.84 a 2.31 enfermeras por cada 1,000 habitantes, cobertura media; de 2.32 a 2.67 enfermeras por 1,000 habitantes, cobertura media alta; y finalmente, entre 2.68 y hasta 3.63 enfermeras por 1,000 habitantes, cobertura alta.

Posteriormente, con las estadísticas de mortalidad infantil, perinatal, materna y en menores de cinco años por entidad federativa y utilizando el método de priorización de componentes principales se construyó el IGM. Con la finalidad de hacer comparables las diferentes tasas de mortalidad, todas se calcularon con factor 1,000.

Tomando como variable dependiente la mortalidad y como variable independiente el ICE se calcularon frecuencias simples y relativas y se hicieron los cuadros y gráficos correspondientes. Finalmente, se realizó un ejercicio de regresión lineal simple.

\section{Resultados}

\section{Cobertura del personal de enfermería}

En México, el personal de enfermería representa más del $50 \%$ del total de personal de salud, hasta el año 2007 se tenían registradas un total de 223,081 enfermeras, para aten- der a una población de 105,790,725 habitantes siendo el indicador de enfermeras por 1, 000 habitantes de 2.11 para la población total. ${ }^{15,16}$

La distribución del personal de enfermería por entidad federativa, el índice de cobertura de servicios de enfermería por cada 1,000 habitantes y el lugar que ocupa cada Estado se aprecia en el cuadro 1.

A partir del ICE y utilizando una distribución cuartilar, se agrupó al total de las Entidades Federativas de la República Mexicana, quedando de la siguiente manera: a) cuartil 1, estados con cobertura menor a 1.83 enfermeras por 1,000 habitantes, fueron San Luis Potosí, Baja California, Guerrero, Querétaro, Guanajuato, Oaxaca, Michoacán, Quintana Roo, Puebla, Chiapas y el Estado de México; b) cuartil 2, estados con cobertura entre 1.8 y 2.31 enfermeras por 1,000 habitantes, incluyó a Yucatán, Nuevo León, Jalisco, Chihuahua, Morelos, Hidalgo, Zacatecas, Veracruz y Tlaxcala; c) cuartil 3, estados con cobertura entre 2.32 y 2.67 enfermeras por 1,000 habitantes, agrupó a Campeche, Nayarit, Coahuila, Sonora, Sinaloa, Tamaulipas y Durango; d) cuartil 4, estados con cobertura mayor o igual a 2.68 enfermeras por 1,000 habitantes concentró al Distrito Federal, Colima, Baja California Sur, Tabasco y Aguascalientes (ver cuadro 2 , gráfica 1 y figura 1 ).

\section{Descripción de la mortalidad}

La mortalidad infantil en México por 1,000 NVR (Nacidos Vivos Registrados), presenta una tasa nacional de 15.4. Las entidades más afectadas son: Estado de México 20.6, Tlaxcala 20.9 y Puebla 23.3; las tasas más bajas se presentan en: Nayarit 8.1, Campeche 8.7 y Sinaloa 9.5 de- 
funciones en niños de uno a doce meses de edad por cada 1,000 NVR.

La mortalidad perinatal por cada 1,000 NV (Nacidos Vivos), presenta una tasa nacional de 21.3. Los estados con tasas más alta son: Estado de México 25.6, Puebla 28.2 y el Distrito Federal 30.8; las tasas más bajas se reportaron en: Sinaloa 6.1, Guerrero 8.6 y Nayarit 9.9 decesos por cada $1,000 \mathrm{NV}$.

La mortalidad materna por cada 1,000 NV, presenta una tasa nacional de 0.556 . Los estados que presentan mayor problema son: Baja California Sur 0.834, Guerrero 0.977 y Oaxaca 1.02; las entidades federativas con menor problema son: Aguascalientes 0.175, Colima 0.197 y Campeche 0.210 muertes maternas por cada $1,000 \mathrm{NV}$.

La mortalidad en menores de cinco años por cada 1,000 nacimientos estimados, presenta una tasa nacional de 18.4. Los estados mayormente afectados son: Oaxaca 23.5, Chiapas 25.8 y Guerrero 26.1; las entidades con menor problema son: Nuevo León 13.4, Distrito Federal 14.5 y los estados de Baja California y Coahuila 15.0 cada uno (ver cuadro 3).

\section{Índice Global de Mortalidad e Índice de Cobertura de Enfermería}

Al realizar la prueba estadística de regresión lineal simple en donde la variable dependiente fue el IGM y la variable independiente el ICE, se encontró un valor de Beta de -.472 y un valor de P de .000 lo cual es indicador de que existe significancia estadística y se interpreta que por cada punto porcentual que se incrementa en el ICE disminuye el IGM en -.472, ésta situación se observa claramente en la gráfica de dispersión con línea de tendencia (ver cuadro 4 y gráfico 2).

De acuerdo con el análisis de regresión lineal simple por nivel de cobertura se observa la misma tendencia que en la regresión para el nivel nacional en lo que respecta a cobertura baja, media y media alta, en estos tres subgrupos es evidente que al incrementar el ICE disminuye el IGM, incluso el coeficiente de determinación $(R)$ tiene una tendencia de incremento (cobertura baja 2052; cobertura media .3257; y cobertura media alta .5083). Sin embargo, esta tendencia se revierte en el caso de la cobertura alta (ver gráficas 3 a 6).

\section{Discusıón}

En este trabajo el elemento central fue buscar la relación de causalidad entre el ICE y el IGM, al respecto, es conveniente hacer algunas precisiones.

La salud es un requisito imprescindible para desarrollar las capacidades de las personas en todos los ámbitos de la vida. ${ }^{6}$ Indiscutiblemente, la muerte, es un hecho inherente a lal vida, pero el cuidado a la salud que el personal de enfermería otorgue a individuos, familias, comunidades y colectivos sociales es un coadyuvante no sólo para procurar y restablecer la salud, si no también, para lograr que la muerte ocurra de manera tardía, en un entorno confortable y preferentemente, que ésta ocurra por causas naturales.

En adhesión al ICE, del cual México forma parte, las principales funciones que el personal de enfermería debe de procurar a las personas que atiende son la defensa de los derechos de los pacientes, la procuración de entornos seguros para la atención, la seguridad del paciente y la calidad de la atención, entre otras, ${ }^{17}$ de acuerdo con datos oficiales del SNS, al hacer el cálculo general de cobertura de enfermería, resulta que nuestro país se ubica en un nivel considerado bajo criterios internacionales como de "Baja Cobertura". Por consiguiente, la pregunta obligada sería ¿Cómo se pretende tener seguridad y calidad en los procesos de atención de enfermería cuando se tiene baja cobertura?

Especialistas del Banco Mundial afirman que "Las enfermeras proporcionan la mayoría de la atención incluida en el paquete básico de servicios de salud pública y clínica. En los servicios clínicos, ellas desempeñan un papel fundamental, dado el impacto de su trabajo en la calidad, eficiencia y eficacia de la atención, que proporcionan las 24 horas al día los 365 días al año,18 por tal razón, es de esperarse que los niveles de salud de la población, estén vinculados al trabajo de enfermería. Por lo tanto, los resultados de este trabajo son concluyentes, las cifras muestran que cuando aumenta el ICE disminuye la mortalidad, no obstante, existen otras realidades, que obligadamente deben de plantearse. ¿Éste fenómeno ocurre de la misma manera cuando se trata de población con seguridad social y población sin seguridad social?; o cuando el análisis se hace por nivel académico del personal de enfermería; o por institución de ocurrencia de la defunción. Seguramente, las interrogantes podrían seguir, desde luego, el propósito del trabajo no es ese. Sin embargo, se considera conveniente que el personal de enfermería incursione en actividades de investi- 
gación poco ortodoxas.

Finalmente, dado que el objetivo de este artículo fue "Identificar como impacta el indicador de cobertura de atención de enfermería en la mortalidad infantil, perinatal, materna y en menores de cinco años" consideramos que éste se cumplió satisfactoriamente.

\section{ConcLusiones}

La Reforma del Sector Salud en México, incluye el desarrollo de estrategias para mejorar la cobertura y la calidad en la prestación de los servicios de salud por lo que es necesario el análisis de los recursos humanos y su capacidad en el cumplimiento de metas en salud. Para el análisis de recursos humanos en salud la ops plantea como necesidades imprescindibles la obtención de la información sobre personal necesario para alcanzar la cobertura y calidad suficiente; las metas en calidad de la atención; y la investigación sobre los recursos necesarios para cumplir con los procesos establecidos.

En este sentido, el estudio de la mortalidad temprana y/o evitable y su relación con la cobertura de enfermería además de cumplir con lo establecido por la OPS, ayuda a la mejor comprensión del impacto final que tiene el profesional de enfermería en el perfil de mortalidad, por lo que es necesario que en la evaluación del desempeño de la profesión de enfermería se evalúen en primera instancia los recursos para la atención como parte esencial de los indicadores de estructura, ya que cuando éstos son suficientes y de alta calidad se pueden proporcionar mejores servicios a la población que se atiende.

En países desarrollados se ha demostrado el impacto que tiene la falta de personal cualificado de enfermería en la evolución de los pacientes. Ante esta situación tan evidente en nuestro país, misma que se ratifica con estadísticas oficiales. Es necesario continuar demostrando de manera contundente con resultados de investigación verídicos, demostrables, comprobables y repetibles; la gran cantidad de ventajas que se tienen al contar con suficiente personal cualificado para la atención a la salud.

La construcción del IGM realizado a partir del método de componentes principales permitió contar con un indicador de valor numérico absoluto que refleja claramente la magnitud de las muertes a edades tempranas como es el caso de la mortalidad perinatal, infantil y en menores de un año; así como las que ocurren en mujeres en periodos maternos, mismos que por ser procesos fisiológicos no deberían ser fatales. Éste índice, permitió priorizar y comparar de manera objetiva la mortalidad de cada estado al interior de toda la República Mexicana.

En cuanto a la cobertura del personal de enfermería, si bien el indicador nacional es de 2.11 por cada 1,000 habitantes, la realidad es que cada entidad federativa presenta una cobertura distinta, el análisis cuartilar demuestra que once estados tienen entre 1.20 y 1.83 enfermeras por 1,000 habitantes, por lo que se encuentran en un bajo nivel de cobertura, nueve presentan un nivel medio, ya que cuentan de 1.84 a 2.31 enfermeras por cada 1,000 habitantes; siete entidades se ubican en nivel medio alto ya que tienen de 2.32 a 2.67 enfermeras por 1,000 habitantes; y finalmente, cinco estados están en un nivel alto por tener entre 2.68 hasta 3.63 enfermeras por 1,000 habitantes.

El ejercicio de regresión lineal simple a nivel nacional demostró que existe significancia estadística entre el ICE y el IGM ya que cuando se incrementa el primero, disminuye el segundo. No obstante, éste mismo análisis por nivel de cobertura baja, media y media alta indica que estos tres subgrupos tienen un comportamiento igual al del nivel nacional, no así en los estados con un nivel alto de cobertura, en donde la tendencia es inversa.

Finalmente, se sabe que más de la mitad del personal de salud en México corresponde al área de enfermería, por lo cual es conveniente continuar realizando trabajos de investigación sobre los recursos humanos de enfermería y su impacto en los indicadores de la salud que permitan a los directivos definir políticas y estrategias en materia de recursos de enfermería relativos a dotación, formación, capacitación y retención.

\section{Notas}

* El Índice Global de Mortalidad se construyó tomando como base la estructura conceptual, matemática y metodológica que Sergio Camposortega Cruz utilizó para la construcción del Îndice de Bienes$\operatorname{tar}$ Social.

\section{REFERENCIAS BIBLIOGRÁFICAS}

1 Organización Panamericana de la Salud. Dotación de recursos humanos en salud: nuevas perspectivas de análisis. Washington D. C. 2005. p. 151.

2 Secretaría de Salud México. Dirección General de Información en Salud. Sistema Nacional de In- 
formación en Salud. Mortalidad: Documento Técnico sobre Mortalidad. Consultado el 29 de abril de 2010. Disponible en: http://sinais.salud.gob.mx/ descargas/pdf/ morttec.pdf.

3 Kérouac S, Pepin J, Ducharme F, Duquette A y Major F. El pensamiento enfermero. Barcelona, España: Ed. Masson; 1996. p 60.

4 Consejo Internacional de Enfermeras. La definición de enfermería. Consultado el 26 de abril de 2010. Dinponible en: http://www.icn. ch/es/about-icn/icn-definition-ofnursing/.

5 Donabedian A. Los espacios de la salud: Aspectos fundamentales de la organización de la atención médica. México: Fondo de Cultura Económica; 1988. p 255.

6 De la Fuente-Ramírez JR, Tapia-Conyer R y Lezana-Fernández MA. La información en salud. México: McGraw-Hill Interamericana; 2002. p 26.

7 Zárate Grajales RA y Salcedo Álvarez RA. La migración, un riesgo potencial para la escasez de enfermeras en México. En: Campos Covarrubias G, Compilador. Educación y salud en los migrantes México - Estados Unidos. México: Miguel Ángel Porrúa; 2009.p. 125-37.

8 Lang TA, Hodge M, Olson V, Romano PS, Kravitz RL. Nurse-patient ratios: a systematic review on the effects of nurse staffing on patient, nurse employee, and hospital outcomes. J Nurs Adm. 2004; 34 (7-8): 326-37.

9 Aiken L, Clarke P, Sloane P, Sochalski J. Silber J. Hospital nurse staffing and patient mortality, nurse burnot and job dissatisfaction. JAMA. 2002; 288 (16): 1987-93.

10 Organización Panamericana de la Salud. Indicadores de salud: Ele- mentos básicos para el análisis de la situación de salud. Boletín Epidemiológico 2001; 22 (4): 1-5.

11 Instituto Nacional de Estadística Geografía e Informática. Documentos conceptuales y metodológicos. Consultado 3 de mayo de 2010. Disponible en: http://www. inegi.org.mx/est/contenidos/espanol/rutinas/glogen/default.aspx?t $=$ RCMORG\&s $=$ est $\& c=11169$.

12 Tapia-Conyer R. El manual de salud pública. Vol. 4. México: Intersistemas; 2003. p 632.

13 Galbiati J. Componentes principales. www.jorgegalbiati.cl/ mayo_06/Componentes.pdf. Recuperado el 22 de abril de 2010.

14. Campos ortega-Cruz S. Población, bienestar y territorio en el Estado de Hidalgo, 1960-1990. México: Universidad Autónoma del Estado de Hidalgo. Colección Sociedad y Pensamiento; 1997. pp 43-49.
15 Secretaría de Salud México. Dirección General de Información en Salud. Sistema Nacional de Información en Salud. Situación de salud en México 2007: Indicadores básicos. pp 1-12.

16 Secretaría de Salud México. Dirección General de Información en Salud. Sistema Nacional de Información en Salud. Recursos físicos, materiales y humanos. Boletín de información estadística 2007; 1(27): 72-85.

17 Consejo Internacional de Enfermeras. Las funciones de enfermería. Consultado 4 de mayo de 2010. Disponible en: http://www.icn. ch/es/about-icn/icn-definition-ofnursing/.

18 Banco Mundial. Informe Anual del Banco Mundial 1993: "Invertir en Salud". Washington D. C., USA. 1993.

\section{Figura 1}

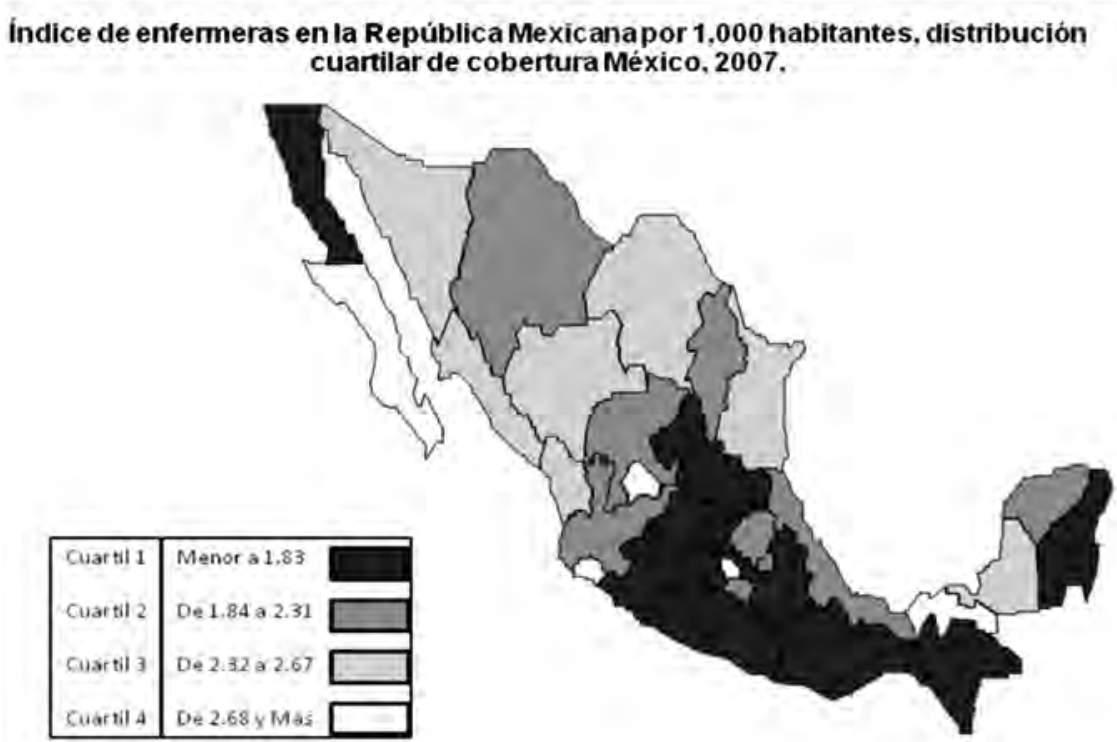

Fuente: 55A. SINAIS. Boletin 27. Menco, 2007 
Cobertura de Recursos Humanos de Enfermería por 1,000 habitantes

Gráfica 1

por Entidad Federativa. México, 2007

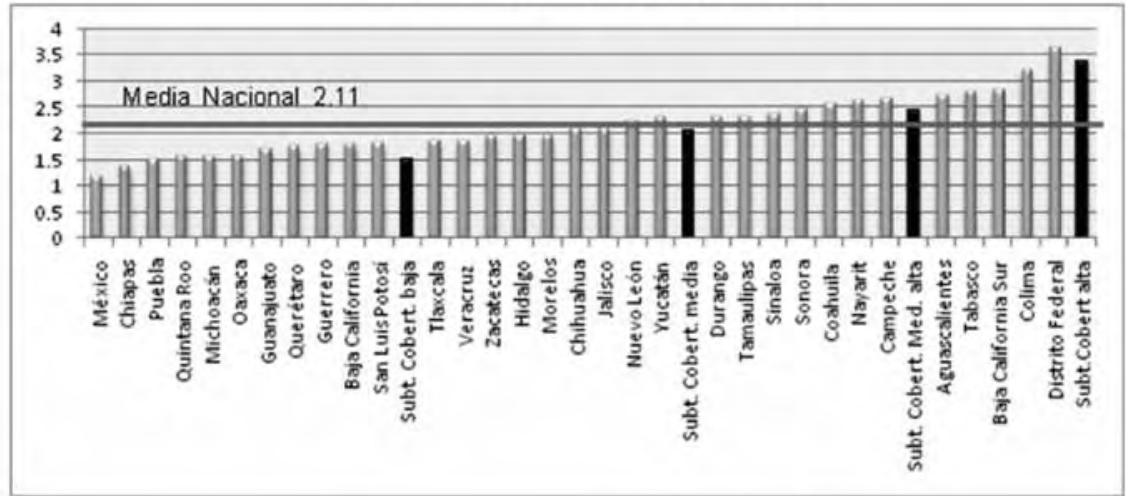

\section{Gráfica 2}

Fuente: Datos del estudio

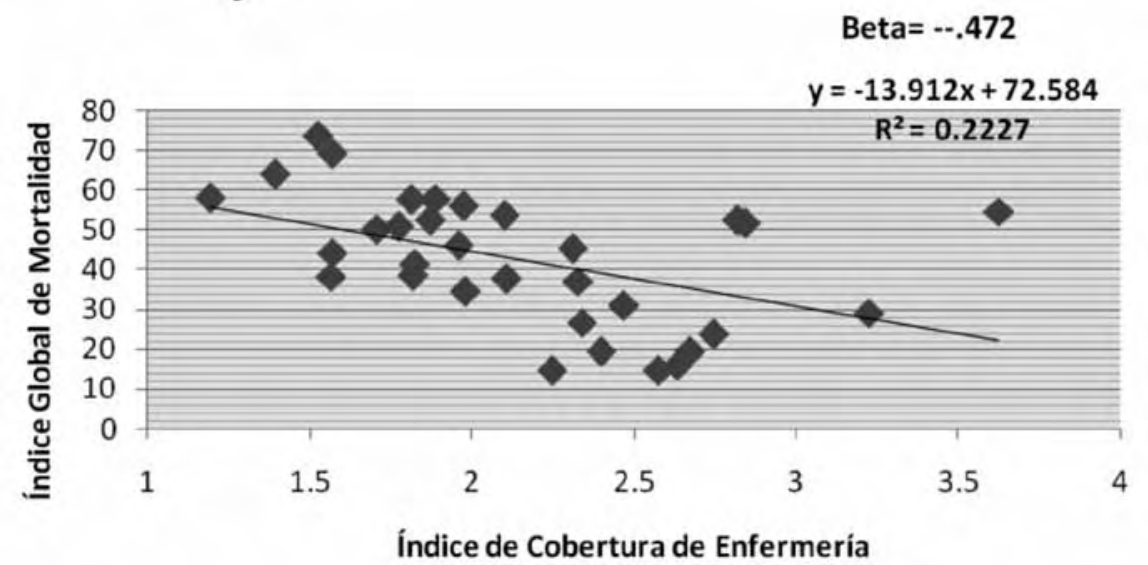

Gráficas 3 a 6 . Regresión lineal del Índice Global de Mortalidad y el Índice de Cobertura de Enfermería por subgrupos de cobertura. México, 2007

Gráfica 3

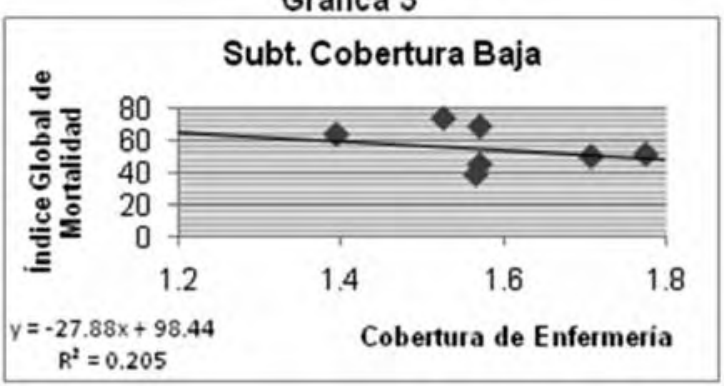

Gráfica 5

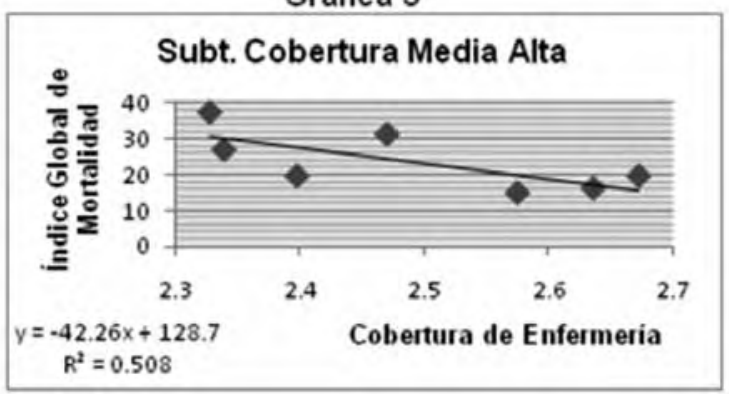

Gräfica 4

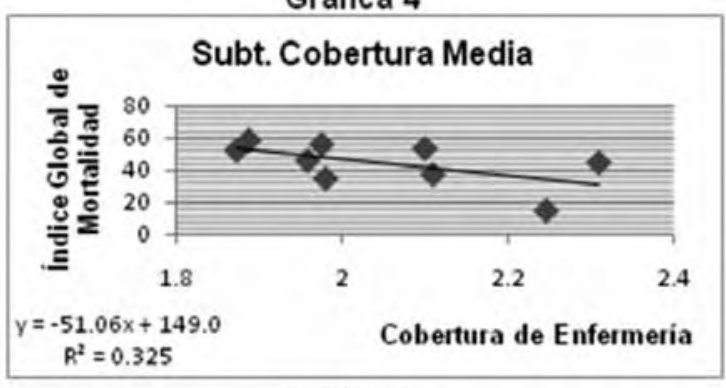

Gráfica 6

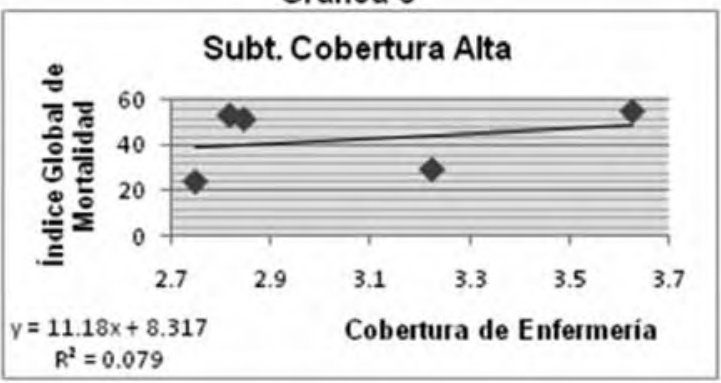

Fuente: Datos del estudio 


\section{Cuadro 1 - Distribución de Recursos Humanos de Enfermería por Entidad Federativa, índice por 1,000 habitantes y lugar en cobertura. México, 2007}

\begin{tabular}{|c|c|c|c|c|c|}
\hline$N^{\circ}$ & $\begin{array}{l}\text { Entidad } \\
\text { Federativa }\end{array}$ & Población & Enfermeras & $\begin{array}{c}\text { Índice } \\
\text { por 1,000 } \\
\text { Habitantes. }\end{array}$ & $\begin{array}{l}\text { Lugar } \\
\text { en } \\
\text { cobertura }\end{array}$ \\
\hline 1 & Aguascalientes & $1,106,319$ & 3,040 & 2.75 & $5^{\circ}$ \\
\hline 2 & Baja California & $2,993,422$ & 5,453 & 1.82 & $23^{\circ}$ \\
\hline 3 & Baja California Sur & 537,586 & 1,530 & 2.85 & $3^{\circ}$ \\
\hline 4 & Campeche & 777,506 & 2,078 & 2.67 & $6^{\circ}$ \\
\hline 5 & Coahuila & $2,573,950$ & 6,628 & 2.58 & $8^{\circ}$ \\
\hline 6 & Colima & 585,429 & 1,889 & 3.23 & $2^{\circ}$ \\
\hline 7 & Chiapas & $4,411,808$ & 6,154 & 1.39 & $31^{\circ}$ \\
\hline 8 & Chihuahua & $3,326,882$ & 6,989 & 2.10 & $16^{\circ}$ \\
\hline 9 & Distrito Federal & $8,829,423$ & 32,013 & 3.63 & $1^{\circ}$ \\
\hline 10 & Durango & $1,538,251$ & 3,581 & 2.33 & $12^{\circ}$ \\
\hline 11 & Guanajuato & $4,995,325$ & 8,534 & 1.71 & $26^{\circ}$ \\
\hline 12 & Guerrero & $3,149,703$ & 5,718 & 1.82 & $24^{\circ}$ \\
\hline 13 & Hidalgo & 2,396,201 & 4,735 & 1.98 & $18^{\circ}$ \\
\hline 14 & Jalisco & $6,903,114$ & 14,564 & 2.11 & $15^{\circ}$ \\
\hline 15 & México & $14,435,284$ & 17,266 & 1.20 & $32^{\circ}$ \\
\hline 16 & Michoacán & $3,991,189$ & 6,273 & 1.57 & $28^{\circ}$ \\
\hline 17 & Morelos & $1,648,463$ & 3,266 & 1.98 & $17^{\circ}$ \\
\hline 18 & Nayarit & 964,285 & 2,542 & 2.64 & $7^{\circ}$ \\
\hline 19 & Nuevo León & $4,337,085$ & 9,748 & 2.25 & $14^{\circ}$ \\
\hline 20 & Oaxaca & $3,553,070$ & 5,586 & 1.57 & $27^{\circ}$ \\
\hline 21 & Puebla & $5,538,621$ & 8,458 & 1.53 & $30^{\circ}$ \\
\hline 22 & Querétaro & $1,659,431$ & 2,945 & 1.77 & $25^{\circ}$ \\
\hline 23 & Quintana Roo & $1,220,891$ & 1,913 & 1.57 & $29^{\circ}$ \\
\hline 24 & San Luis Potosí & $2,461,624$ & 4,493 & 1.83 & $22^{\circ}$ \\
\hline 25 & Sinaloa & $2,643,536$ & 6,339 & 2.40 & $10^{\circ}$ \\
\hline 26 & Sonora & $2,463,707$ & 6,086 & 2.47 & $9^{\circ}$ \\
\hline 27 & Tabasco & $2,029,035$ & 5,722 & 2.82 & $4^{\circ}$ \\
\hline 28 & Tamaulipas & $3,116,054$ & 7,289 & 2.34 & $11^{\circ}$ \\
\hline 29 & Tlaxcala & $1,104,580$ & 2,071 & 1.87 & $21^{\circ}$ \\
\hline 30 & Veracruz & $7,242,133$ & 13,669 & 1.89 & $20^{\circ}$ \\
\hline 31 & Yucatán & $1,874,235$ & 4,330 & 2.31 & $13^{\circ}$ \\
\hline 32 & Zacatecas & $1,382,583$ & 2,708 & 1.96 & $19^{\circ}$ \\
\hline \multicolumn{2}{|c|}{ TOTAL } & $105,790,725$ & 223,081 & 2.11 & N/A \\
\hline
\end{tabular}




\section{Enfermería Universitaria}

\section{Cuadro 2. Distribución cuartilar e Índice de Cobertura de Enfermería por 1,000 habitantes en la República Mexicana. México, 2007}

\begin{tabular}{|c|c|c|c|c|}
\hline Entidad Federativa & $\begin{array}{l}\text { Înd. Enf/Pob. } \\
\text { Por 1,000 Hab. }\end{array}$ & Porcentaje & $\begin{array}{l}\text { Porcentaje } \\
\text { Acumulado }\end{array}$ & Cuartil \\
\hline Estado de México & 1.20 & 1.74 & 1.74 & \\
\hline Chiapas & 1.39 & 2.02 & 3.76 & \\
\hline Puebla & 1.53 & 2.22 & 5.98 & \\
\hline Quintana Roo & 1.57 & 2.27 & 8.25 & \\
\hline Michoacán & 1.57 & 2.28 & 10.53 & \\
\hline Oaxaca & 1.57 & 2.28 & 12.82 & \\
\hline Guanajuato & 1.71 & 2.48 & 15.30 & \\
\hline Querétaro & 1.77 & 2.58 & 17.87 & \\
\hline Guerrero & 1.82 & 2.63 & 20.51 & \\
\hline Baja California & 1.82 & 2.64 & 23.15 & \\
\hline San Luis Potosí & 1.83 & 2.65 & 25.80 & Cuartil 1 \\
\hline Tlaxcala & 1.87 & 2.72 & 28.52 & \\
\hline Veracruz & 1.89 & 2.74 & 31.26 & \\
\hline Zacatecas & 1.96 & 2.84 & 34.10 & \\
\hline Hidalgo & 1.98 & 2.87 & 36.97 & \\
\hline Morelos & 1.98 & 2.88 & 39.84 & \\
\hline Chihuahua & 2.10 & 3.05 & 42.89 & \\
\hline Jalisco & 2.11 & 3.06 & 45.95 & \\
\hline Nuevo León & 2.25 & 3.26 & 49.21 & \\
\hline Yucatán & 2.31 & 3.35 & 52.57 & Cuartil 2 \\
\hline Durango & 2.33 & 3.38 & 55.95 & \\
\hline Tamaulipas & 2.34 & 3.39 & 59.34 & \\
\hline Sinaloa & 2.40 & 3.48 & 62.82 & \\
\hline Sonora & 2.47 & 3.58 & 66.40 & \\
\hline Coahuila & 2.58 & 3.74 & 70.14 & \\
\hline Nayarit & 2.64 & 3.83 & 73.97 & \\
\hline Campeche & 2.67 & 3.88 & 77.85 & Cuartil 3 \\
\hline Aguascalientes & 2.75 & 3.99 & 81.83 & \\
\hline Tabasco & 2.82 & 4.09 & 85.93 & \\
\hline Baja California Sur & 2.85 & 4.13 & 90.06 & \\
\hline Colima & 3.23 & 4.68 & 94.74 & \\
\hline Distrito Federal & 3.63 & 5.26 & 100.00 & Cuartil 4 \\
\hline
\end{tabular}

Fuente: Datos del estudio 
Cuadro 3 - Mortalidad infantil, perinatal, materna y en menores de cinco años en la República Mexicana. México, 2007

\begin{tabular}{|c|c|c|c|c|}
\hline \multirow{2}{*}{ Entidad federativa } & \multicolumn{4}{|c|}{ Mortalidad por 1,000 habitantes o NVR según sea el caso } \\
\hline & Infantil & Perinatal & Materna & $<5$ años \\
\hline Aguascalientes & 10.8 & 20.8 & 0.175 & 16.6 \\
\hline Baja California & 16.2 & 23.5 & 0.401 & 15 \\
\hline Baja California Sur & 15.8 & 23.5 & 0.834 & 15.4 \\
\hline Campeche & 8.7 & 13.4 & 0.21 & 19.2 \\
\hline Coahuila & 11.6 & 11.2 & 0.27 & 15 \\
\hline Colima & 13.4 & 23.2 & 0.197 & 15.7 \\
\hline Chiapas & 13.2 & 18.4 & 0.815 & 25.8 \\
\hline Chihuahua & 17.1 & 22.8 & 0.749 & 16.8 \\
\hline Distrito Federal & 17.1 & 30.8 & 0.681 & 14.5 \\
\hline Durango & 11.9 & 17.7 & 0.469 & 19.4 \\
\hline Guanajuato & 16.4 & 24.3 & 0.396 & 19.9 \\
\hline Guerrero & 12.1 & 8.6 & 0.977 & 26.1 \\
\hline Hidalgo & 15.3 & 23.3 & 0.659 & 20.4 \\
\hline Jalisco & 14.5 & 22.7 & 0.354 & 16.9 \\
\hline México & 20.6 & 25.6 & 0.54 & 17.8 \\
\hline Michoacán & 13.2 & 17.7 & 0.515 & 21.1 \\
\hline Morelos & 13.5 & 16.9 & 0.52 & 16.7 \\
\hline Nayarit & 8.1 & 9.9 & 0.29 & 18.6 \\
\hline Nuevo León & 10.9 & 16.9 & 0.221 & 13.4 \\
\hline Oaxaca & 14.9 & 19.7 & 1.02 & 23.5 \\
\hline Puebla & 23.3 & 28.2 & 0.514 & 22.1 \\
\hline Querétaro & 17 & 22.8 & 0.532 & 18.6 \\
\hline Quintana Roo & 12.3 & 21.5 & 0.568 & 16.5 \\
\hline San Luis Potosí & 11.2 & 17.8 & 0.619 & 19.7 \\
\hline Sinaloa & 9.5 & 6.1 & 0.538 & 17.5 \\
\hline Sonora & 13 & 18.5 & 0.444 & 15.7 \\
\hline Tabasco & 14.7 & 23.6 & 0.591 & 20 \\
\hline Tamaulipas & 13.3 & 14.5 & 0.441 & 15.4 \\
\hline Tlaxcala & 20.9 & 21.8 & 0.315 & 19.9 \\
\hline Veracruz & 14 & 20.7 & 0.756 & 22 \\
\hline Yucatán & 12.3 & 22.3 & 0.605 & 18.8 \\
\hline Zacatecas & 15 & 21 & 0.374 & 21 \\
\hline Nacional & 15.4 & 21.3 & 0.556 & 18.4 \\
\hline
\end{tabular}




\section{Cuadro 4 - Cobertura de Atención de Enfermería e Índice Global de Mortalidad en la República Mexicana. México, 2007}

\begin{tabular}{|c|c|c|}
\hline Entidad federativa & $\begin{array}{l}\text { Îndice de Cobertura } \\
\text { de Enfermería }\end{array}$ & $\begin{array}{l}\text { Índice Global } \\
\text { de Mortalidad }\end{array}$ \\
\hline México & 1.20 & 58.207 \\
\hline Chiapas & 1.39 & 64.126 \\
\hline Puebla & 1.53 & 73.777 \\
\hline Quintana Roo & 1.57 & 38.433 \\
\hline Michoacán & 1.57 & 44.412 \\
\hline Oaxaca & 1.57 & 69.346 \\
\hline Guanajuato & 1.71 & 50.249 \\
\hline Querétaro & 1.77 & 50.939 \\
\hline Guerrero & 1.82 & 57.837 \\
\hline Baja California & 1.82 & 38.698 \\
\hline San Luis Potosí & 1.83 & 41.284 \\
\hline Subtotal de cobertura baja & 1.50 & N/A \\
\hline Tlaxcala & 1.87 & 52.723 \\
\hline Veracruz & 1.89 & 57.834 \\
\hline Zacatecas & 1.96 & 46.326 \\
\hline Hidalgo & 1.98 & 56.286 \\
\hline Morelos & 1.98 & 34.761 \\
\hline Chihuahua & 2.10 & 53.645 \\
\hline Jalisco & 2.11 & 37.796 \\
\hline Nuevo León & 2.25 & 14.527 \\
\hline Yucatán & 2.31 & 45.294 \\
\hline Subtotal de cobertura media & 2.05 & $\mathrm{~N} / \mathrm{A}$ \\
\hline Durango & 2.33 & 37.249 \\
\hline Tamaulipas & 2.34 & 26.864 \\
\hline Sinaloa & 2.40 & 19.508 \\
\hline Sonora & 2.47 & 31.155 \\
\hline Coahuila & 2.58 & 14.807 \\
\hline Nayarit & 2.64 & 16.085 \\
\hline Campeche & 2.67 & 19.540 \\
\hline Subtotal de cobertura media alta & 2.45 & N/A \\
\hline Aguascalientes & 2.75 & 23.845 \\
\hline Tabasco & 2.82 & 52.729 \\
\hline Baja California Sur & 2.85 & 51.713 \\
\hline Colima & 3.23 & 29.262 \\
\hline Distrito Federal & 3.63 & 54.773 \\
\hline Subtotal de cobertura alta & 3.37 & $\mathrm{~N} / \mathrm{A}$ \\
\hline Gran total & 2.11 & N/A \\
\hline
\end{tabular}

